\section{Secondary variants and human subjects research}

To the Editor: In the article titled "Return of Research Results from Genomic Biobanks: Cost Matters," Bledsoe et al. ${ }^{1}$ have performed a valuable service by outlining their concerns regarding the potential cost to biobanks of policies that favor the return of individual research results to clinical genomics research participants. They correctly characterize the ClinSeq study as one that has an ongoing, direct relationship with the participants and in which the return of results is practical. They contrast ClinSeq to biobank studies in which the data analysts have several degrees of separation from the participants and in which developing the infrastructure to return results could be complex and costly. Their plea is that the relationship of the research participant to the biobank and the costs of returning results are important factors to consider and that applying the ClinSeq model to all biobanks is impractical and could harm the larger research enterprise. These are important concerns, and research to quantify the cost of returning results in a range of research designs is sorely needed.

Yet Bledsoe et al. ${ }^{1}$ seem to discourage any return of results in research involving biobanks, except when the biobank maintains the kind of direct involvement with participants that ClinSeq has. This may have the unintended consequence of incentivizing researchers to structure human genomics research studies as remote biobank-style studies to reduce costs and discourage direct engagement with research participants. I would argue that it is undesirable if this incentive were to be broadly realized, as our field needs to move toward more, not less, participant engagement. A reasonable question could be raised whether our field would benefit more from a larger number of less expensive, narrowly defined biobank studies with no participant engagement or a smaller number of more expensive studies with high degrees of ongoing participant participation, iterative phenotyping, and return of results.

I recognize that the law of unintended consequences operates at many levels in human subjects research-whenever we increase standards and human subjects protections, we incur increased costs and risk delays in discovery. Yet, recognizing what we owe back to research participants is a crucial part of ethical research. Bledsoe et al. ${ }^{1}$ recognized the importance of returning some results, those that are analytically and clinically valid, confirmed in a Clinical Laboratory Improvement Amendments-certified lab, and have serious consequences for participants. We have to consider how best to identify medically important results and offer them back to participants in the range of research models we have, including those involving biobanks.
Our field cannot go to the extreme of returning nothing, whether a biobank is involved in a protocol or not. We need to move toward robust and ongoing participant engagement, for scientific reasons, out of respect for our participants, and to maintain the public support of the research enterprise. I propose to translational genomics researchers the following thought experiment: imagine yourself in a room full of the participants at the close of your study, and your assignment is to describe to them the kinds of medically useful data in your data set and then justify why you gave none of it back to them. I can't do it.

\section{ACKNOWLEDGMENTS}

The author is supported by the Intramural Research Program of the National Human Genome Research Institute. The opinions expressed here are his own and do not necessarily reflect the views of any institution to which he is affiliated.

\section{DISCLOSURE}

The author is an uncompensated consultant to, and collaborates with scientists at, the Illumina Corporation as a part of his official duties.

\section{Leslie G. Biesecker, $M D^{1}$}

${ }^{1}$ Genetic Disease Research Branch, National Human Genome Research Institute, National Institutes of Health, Bethesda, Maryland, USA. Correspondence: Leslie G. Biesecker (lesb@mail.nih.gov)

\section{REFERENCE}

1. Bledsoe MJ, Clayton EW, McGuire AL, Grizzle WE, O'Rourke PP, Zeps N. Return of research results from genomic biobanks: cost matters. Genet Med 2012; e-pub ahead of print 30 August 2012.

doi:10.1038/gim.2012.161

\section{Return of results in genomic biobank research: ethics matters}

To the Editor: In "Return of Results from Genomic Biobanks: Cost Matters," Bledsoe et al. ${ }^{1}$ offer critique of a 26-author consensus article published in April's Genetics in Medicine articulating the first full analysis of the challenge posed to biobanks by the debate over return of individual research results (IRRs) and incidental findings (IFs). ${ }^{2}$ They raise important questions concerning the cost to biobanks. As lead author of the April paper, I write to respond.

Bledsoe et al. ${ }^{1}$ argue that our paper suggests responsibilities for biobanks that would impose "unsustainable" cost. Unfortunately, they do not address our own discussion of cost. Our study suggested cutting back on what IRRs and IFs should be returned in part because of cost: "The greater difficulty and cost of biobank return, the lower likelihood 
of benefit with lapse of time, and the reality that some contributors will not have consented to research, justify more restrictive criteria for return in biobank research than primary research." We included an important recommendation urging that "Research and biobank funders and regulators have a crucial role to play in making sure that research and biobank budgets adequately support responsible management of IFs and IRRs." This recommendation can support biobanks as they articulate what resources they need to deal with the return of IRRs and IFs.

The reality is that all research could be done less expensively and faster if we ignored ethical responsibilities to research participants. Ethics costs money and requires effort, starting with informed consent. The mere fact that dealing with the return of IRRs and IFs will take effort and resources is not an argument against it. As yet, there are very few published studies analyzing the cost, especially in the complex context of a biobank research system. We defined that system to include (i) primary research or specimen- and data-collection sites, (ii) the biobank itself, and (iii) secondary researchers analyzing those specimens and data. As we pointed out, IRRs and IFs can arise at all three sites. Quantifying the cost of handling IRRs and IFs will not be easy. Cost will probably vary substantially, depending on the design of the biobank research system and ethical choices made within that system on how to manage IRRs and IFs.

Bledsoe et al. ${ }^{1}$ may overestimate the burden and costs we place on biobanks themselves. We carefully analyzed biobanks as one part of the larger biobank research system. This is because the purpose of biobanks is to aggregate data and specimens to fuel secondary research; biobanks enable the flow of data and specimens through the research system. Bledsoe et al. ${ }^{1}$ incorrectly suggest that we placed on biobanks alone the responsibility to establish criteria for evaluating IFs and IRRs, analyze those findings, reidentify participants, and recontact them to offer the findings. Instead, we urged that biobanks collaborate with primary researchers (or collection sites) and secondary researchers. Specifically, we recommended that biobanks work with both primary and secondary researchers to clarify criteria for evaluating findings but let primary researchers analyze findings arising at their own sites so that biobanks can focus on those arising later in the biobank research system. We called for biobanks to collaborate with primary researchers to decide jointly on how to handle reidentification, and we described different options including the use of a "trusted intermediary." However, once a participant is reidentified, we recommended that biobanks generally leave the task of recontact to primary research sites when those sites have had direct contact with participants. Thus, our recommendations built in flexibility, so that different biobank research systems could allocate responsibilities within the system according to the research realities.

Bledsoe et al. ${ }^{1}$ appear to construe our paper as a "call for the routine evaluation and return of IFs and IRRs from all biobanks." However, our study discussed at length the variety of biobanks and noted that some biobanks cannot return IFs and IRRs because they irretrievably strip identifiers, making return of IFs and IRRs impossible. For those biobanks and biobank research systems retaining the capacity to reidentify and return, we urged the "development of explicit policy on whether IFs and IRRs will be returned." We then recommended that the biobank research system differentiate the limited list of findings that "should" be returned from a potentially broader list that "may" be returned depending on decisions made within the biobank research system. Again, we built in considerable flexibility.

Bledsoe et al. ${ }^{1}$ conclude that the variety of biobanks argues against the kind of broad recommendations we offer and instead requires "case-by-case" analysis. However, the variety of biobanks does not make ethics recommendations futile. The National Cancer Institute's Office of Biorepositories and Biospecimen Research has issued guidelines for biobanks, ${ }^{3}$ and a hefty literature offers ethics recommendations for biobanks, despite their variety. Many biobanks and biobank research systems are already trying to address return of results and looking for guidance. We offered analysis and concrete recommendations to advance the field.

Leaving biobanks and biobank research systems to face the return-of-results challenge one by one with no recommendations to guide them will reduce efficiency and increase costs, as biobanks struggle individually to figure out what to do. It will also do little to advance collective and coordinated consideration of what our research community owes individuals who are generous enough to provide their data and specimens. Presenting the kind of detailed analysis and concrete recommendations we offer in our article is the necessary next step in the real world of genomic research powered by biobanks collecting data and samples. It sets the stage for future refinement of recommendations, as thinking progresses on how to advance research while advancing our collective understanding of ethical responsibilities.

\section{ACKNOWLEDGMENTS}

Preparation of the April article to which Bledsoe et al. ${ }^{1}$ responded was supported by National Institutes of Health/National Human GenomeResearch Institute(NIH/NHGRI) grant2-R01-HG003178 on "Managing Incidental Findings and Research Results in Genomic Biobanks \& Archives" (S.M.W., principal investigator (PI); J.P. Kahn, F. Lawrenz, B. Van Ness, co-investigators). The author's current work on return of results is supported by NIH/ National Cancer Institute (NCI)/NHGRI grant 1-R01-CA154517 (G. Petersen, B. Koenig, S.M.W., PIs) and a Robert Wood Johnson Foundation (RWJF) Investigator Award 69763 (S.M.W., PI).

\section{DISCLOSURE}

The contents of this letter are solely the responsibility of the author and do not necessarily represent the views of the full author group of the April article, NIH, NHGRI, NCI, or RWJF. The author of this letter has received honoraria for lecturing and 
participating in discussion on return of results, including from the Mayo Clinic and Boston Scientific.

Susan M. Wolf, JD

${ }^{1}$ Law School; Medical School; Center for Bioethics; Consortium on Law and Values in Health, Environment and the Life Sciences, University of Minnesota, Minneapolis, Minnesota, USA. Email: Susan M. Wolf (swolf@umn.edu)

\section{REFERENCES}

1. Bledsoe MJ, Clayton EW, McGuire AL, et al. Return of research results from genomic biobanks: cost matters. Genet Med 2012; doi:10.10389/ gim.2012.105.

2. Wolf SM, Crock BN, Van Ness B, et al. Managing incidental findings and research results in genomic research involving biobanks and archived data sets. Genet Med 2012;14:361-384.

3. $\mathrm{NCl}$, Office of Biorepositories and Biospecimen Research. $\mathrm{NCl}$ Best Practices for Biospecimen Resources, 2011. http://biospecimens.cancer.gov/ bestpractices/2011-NCIBestPractices.pdf. Accessed 5 October 2012

doi:10.1038/gim.2012.162

\section{Return of research results from genomic biobanks: a call for data}

To the Editor: We welcome the letters from Susan Wolf ${ }^{1}$ ("Return of Results in Genomic Biobank Research: Ethics Matters") and Les Biesecker, ("Secondary Variants and Human Subjects Research") that comment on our recent paper, "Return of Research Results From Genomic Biobanks: Cost Matters," ${ }^{\prime 3}$ and provide the opportunity to continue the dialogue on this important and complex topic. We agree that ethical responsibilities to research participants cannot be ignored. However, consideration of these ethical responsibilities must include not only arguments favoring return of research results, but also the potential risks to individuals, and the burdens and costs to individuals, the research enterprise, and society as a whole.

In Susan Wolf's letter to the editor, ${ }^{1}$ she points out that the discussion section of their article ${ }^{4}$ addresses cost as a criti$\mathrm{cal}$ issue and maintains that sufficient flexibility for biobanks is provided. Indeed, the article notes the crucial role that research and biobank funders and regulators would have to assume in order to sustain adequate support for the responsible management of incidental findings and research results. The suggestion is made that by using strict criteria for deciding which results to return, biobanks would be able to limit costs. In addition, financial responsibility would not have to rest completely on the bank; investigators could bear some of this responsibility. Although we agree that the discussion section of the article does address costs and provides some flexibility for biobanks, these considerations are not adequately reflected in the recommendations. We are concerned that policy and law makers may reasonably conclude from the way the recommendations of the paper by Wolf et al. ${ }^{4}$ are written that biobanks should be principally responsible for the evaluation of findings and ensuring return of research results from any biobank in which it is possible to reidentify participants. We contend that regardless of who assumes the responsibility, these costs are likely to be substantial in many cases. Although developing strict criteria for the return of research results from some biobanks would directly affect the cost, it is unclear how much savings it would provide given the expense of setting up an extensive infrastructure for evaluating and ensuring appropriate return of findings. Given the current funding constraints on the whole research enterprise, there is a real ethical tension between being able to afford to do the kind of research that leads to tangible benefits for a large number of people versus the need to manage and deliver validated individual research results in a meaningful and ethically appropriate way. We believe that the debate around personal versus community benefit based on real economic evaluation and practicability must also be included in any analysis of the ethical issues of return of research results.

Dr Biesecker's letter to the editor ${ }^{2}$ raises several issues that merit further discussion. He states that in our recent commentary, we appear to discourage any return of results in research involving biobanks except when the biobank maintains the kind of direct involvement with participants as seen in ClinSeq. We, however, do not make this assertion; rather, our reason for mentioning ClinSeq was to use it as an example of a bank that generates primary research results and in which there is direct interaction with participants. These characteristics provide a stark comparison to the $\mathrm{dbGaP}$ model, in which the biobank has no relationship with the participant and in which data are shared with many investigators for secondary research projects. Our goal was to simply emphasize how different models present very different challenges in the discussion of return of results.

Dr Biesecker's letter addresses the importance of participant engagement in any discussion of return of research results. $\mathrm{He}$ raises the question of whether the field would benefit more from a larger number of less expensive, narrowly defined biobank studies with no participant engagement or a smaller number of more expensive studies with high degrees of ongoing participant engagement, iterative phenotyping, and return of results. We agree that it is important to engage research participants in biobanking research through direct interaction whenever it is possible to do so. However, we do not agree that it is ethically required, nor do we think that all biobanking research must be performed using participant engagement models similar to ClinSeq. Biobanks that are established from existing specimens (e.g., pathology archives or specimens from previously collected projects) are also needed, even if it is not possible to reidentify participants/contributors or to provide them with individual research results. Many of these existing collections may be uniquely valuable because of extensive amounts of clinical follow-up data or due to changes in standards of care (e.g., untreated, node-negative breast cancer cases), and they could not be established prospectively today. Furthermore, it is arguable whether participants/contributors must derive personal 
benefit from return of research results to be engaged in the research; there are many other ways of engaging participant/ contributors in biobanking research.

In summary, biobanking must be conducted in an ethically responsible way. However, continued discussion is needed regarding the ethical obligations and practical implementation issues for returning research results from biobanks. Good data are needed on the actual benefits, risks, and burdens of the return of individual findings from research. This is an evolving issue that must be informed not only by advances in the science but also by experience addressing the challenges of incorporating genomic information into the clinic. Recent discussions on this topic raise questions about our ability to manage the return of genomic findings even in the clinic. ${ }^{5}$ Care must be taken so that the return of individual findings generated in research does not get ahead of what is acceptable for return in clinical care. Additional data in these areas will help inform the development of guidance and approaches to this topic that will respect participants/contributors, while advancing important and ethically responsible research. In the meantime, we refer those interested to the forthcoming Australian guidelines, ${ }^{6}$ which take a different approach to this problem and which we believe may provide a clear way forward for the foreseeable future.

\section{ACKNOWLEDGMENTS}

This article was supported by the Department of Veterans Affairs, Veterans Health Administration, Office of Research and Development, Biomedical Laboratory Research and Development Service (M.J.B.), St. John of God HealthCare (N.Z.), and the National Center for Advancing Translational Sciences of the National Institutes of Health under award no. UL1 TR000445 (E.W.C.). The viewpoints expressed herein represent the authors' personal perspectives and should not be taken as representing the official viewpoint or position of the Department of Veterans Affairs or the National Institutes of Health.

\section{DISCLOSURE}

M.J.B. is a program manager for the Department of Veterans Affairs biorepositories and biobanks and is a member of the International Society for Biological and Environmental Repositories (ISBER). N.Z. operates a cancer biobank at St. John of God HealthCare within its Pathology Practice in the Bendat Family Comprehensive Cancer Centre. He is a member of ISBER and the Australasian Biospecimen Network Association. W.E.G. operates tumor banks as part of the Breast, Pancreatic, and Cervical Specialized Programs of Research Excellence at the University of Alabama at Birmingham and the Pulmonary Hypertension Breakthrough Initiative and prospective tissue repositories as part of the Cooperative Human Tissue Network and the Comprehensive Cancer Center and is a member of ISBER. He is a member of the ethics committee of the U54 grant, U54 MSM/TU/ UAB Comprehensive Cancer Center Partnership. E.W.C. has long been involved in the creation, maintenance, and assessment of BioVu and has been studying ethical issues in genetics/genomics research for many years. She was part of the working group on biobanks convened by Professor Wolf but is not an author of its final document because she did not endorse its analysis and conclusions. A.L.M. and P.P.O. declared no conflict of interest. The authors are funded by their affiliated institutions.

Marianna J. Bledsoe, $M A^{1}$, Ellen Wright Clayton, $M D$, JD', Amy L. McGuire, JD, PhD', William E. Grizzle, $\mathrm{MD}, \mathrm{PhD}^{4}$, P. Pearl O'Rourke, $M D^{5}$ and Nikolajs Zeps BSc (Hons), PhD ${ }^{6,7}$

${ }^{1}$ Department of Veterans Affairs, Washington, DC, USA; ${ }^{2}$ Center for Biomedical Ethics and Society, Vanderbilt University, Nashville, Tennessee, USA; ${ }^{3}$ Center for Medical Ethics and Health Policy, Baylor College of Medicine, Houston, Texas, USA; ${ }^{4}$ University of Alabama at Birmingham, Birmingham, Alabama, USA; ${ }^{5}$ Partners HealthCare, Boston, Massachusetts, USA; ${ }^{6}$ St John of God HealthCare, Subiaco, Australia; ${ }^{7}$ School of Surgery and School of Pathology and Laboratory Medicine, The University of Western Australia, Crawley, Perth, Western Australia. E-mail: Marianna J. Bledsoe marianna.bledsoe@va.gov

\section{REFERENCES}

1. Wolf SM. Return of results in genomic biobank research: ethics matters. Genet Med 2012; doi:10.1038/gim.2012.162.

2. Biesecker LG. Secondary variants and human subjects research. Genet Med 2012; doi:10.1038/gim.2012.161.

3. Bledsoe MJ, Clayton EW, McGuire AL, et al. Return of research results from genomic biobanks: cost matters. Genet Med 2012; doi:10.1038/ gim.2012.105.

4. Wolf SM, Crock BN, Van Ness B, et al. Managing incidental findings and research results in genomic research involving biobanks and archived data sets. Genet Med 2012;14:361-384

5. Biesecker LG, Burke W, Kohane I, Plon SE, Zimmern R. Next-generation sequencing in the clinic: are we ready? Nat Rev Genet 2012;13:818-824.

6. Australian Government. National Health and Medical Research Council. Proposed Revisions to the National Statement on Ethical Conduct in Human Research 2007. Chapter 3.4-Human biospecimens, September 2012. http://consultations.nhmrc.gov.au/files/consultations/drafts/ consultationnationalstatementethicalconduct120904.pdf. Accessed 22 October 2012.

doi:10.1038/gim.2012.163 